\title{
BMJ Open Effectiveness of a computerised system of patient education in clinical practice: a longitudinal nested cohort study
}

\author{
Chia-Hsien Su, ${ }^{1,2}$ Tsai-Chung Li, ${ }^{3}$ Der-Yang Cho, ${ }^{4,5}$ Wei-Fen Ma, ${ }^{6,7}$ \\ Yu-Shan Chang, ${ }^{1,6}$ Tsung-Han Lee, ${ }^{8}$ Li-Chi Huang ${ }^{6,7}$
}

To cite: Su C-H, Li T-C, Cho D-Y, et al. Effectiveness of a computerised system of patient education in clinical practice: a longitudinal nested cohort study. BMJ Open 2018;8:e020621. doi:10.1136/ bmjopen-2017-020621

- Prepublication history for this paper is available online. To view these files, please visit the journal online (http://dx.doi. org/10.1136/bmjopen-2017020621).

C-HS and Y-SC contributed equally.

Received 16 November 2017

Revised 9 March 2018

Accepted 9 March 2018

Check for updates

${ }^{1}$ Department of Public Health, China Medical University, Taichung, Taiwan

${ }^{2}$ Nursing, New Taipei City Hospital, Taipei, Taiwan

${ }^{3}$ Graduate Institute of

Biostatistics, China Medical

University, Taichung, Taiwan

${ }^{4}$ Graduate Institute of Biomedical Sciences, China Medical

University, Taichung, Taiwan

${ }^{5}$ Department of Neurosurgery,

China Medical University

Hospital, Taichung, Taiwan

${ }^{6}$ Department of Nursing, China

Medical University Hospital,

Taichung, Taiwan

${ }^{7}$ School of Nursing, China

Medical University, Taichung,

Taiwan

${ }^{8}$ Information Technology Office,

China Medical University

Hospital, Taichung, Taiwan

Correspondence to

Professor Li-Chi Huang;

lichi@mail.cmu.edu.tw

\section{ABSTRACT}

Introduction Developing electronic health record information systems is an international trend for promoting the integration of health information and enhancing the quality of medical services. Patient education is a frequent intervention in nursing care, and recording the amount and quality of patient education have become essential in the nursing record. The aims of this study are (1): to develop a high-quality Patient Education Assessment and Description Record System (PEADRS) in the electronic medical record (2); to examine the effectiveness of the PEADRS on documentation and nurses' satisfaction (3); to facilitate communication and cooperation between professionals. Methods and analysis A quasi-experimental design and random sampling will be used. The participants are nurses who are involved in patient education by using traditional record or the PEADRS at a medical centre. A prospective Iongitudinal nested cohort study will be conducted to compare the effectiveness of the PEADRS, including (1): the length of nursing documentation (2); satisfaction with using the PEADRS; and (3) the benefit to professional cooperation.

Ethics and dissemination Patient privacy will be protected according to Electronic Medical Record Management Practices of the hospital. This study develops a patient education digital record system, which would profit the quality of clinical practice in health education. The results will be published in peer-reviewed journals and will be presented at scientific conferences.

\section{INTRODUCTION}

The use of computerised systems in healthcare has been growing globally. Electronic health records are computerised systems that allow storage, retrieval and sharing of information among professionals. ${ }^{1}$ Electronic health record information systems standardise specific content, including documentation standards, storage, labelling and certification. Such records promote the integration of health information to enhance the quality of health services. $^{2}$

Paper copies of health records require large amounts of storage space. In addition, they increase the operating and personnel costs of hospitals. Health records produced

\section{Strengths and limitations of this study}

- We develop a Patient Education Assessment and Description Record System (PEADRS) that is first linkage of patient education into nursing records digitally in the electronic medical information system.

- $\triangle$ prospective longitudinal nested cohort design was chosen to evaluate the effectiveness of the PEADRS in the quality of patient education and professionals' cooperation.

- A potential limitation of the study is the small sample size, when patient education is not done for invasive procedures or examinations and operation that may affect the generalisability and external validity of the result of study.

and stored in electronic format may preclude writing and storage of paper copies. Electronic records make access to health records faster and enhance the quality of service and improve the efficiency of medical resources. ${ }^{34}$ To fulfil the requirements of the Electronic Medical Record Adoption Model Stage 6 Award Survey in April 2017, our hospital had to achieve a near-paperless environment that harnessed technology to support optimised patient care. ${ }^{5}$

Patient education is an important nursing intervention $^{6}$ and an important aspect of the nursing documentation. Patient education has been viewed as an important part of providing quality healthcare that respects and safeguards the rights of patients. Research has shown that patient education provides knowledge regarding healthcare, establishes healthy behaviour, shortens hospitalisation and reduces rehospitalisation. ${ }^{7}$ Patient education is foundational for improving compliance with and success of patient engagement initiatives. ${ }^{8-10}$ Patient education affects the patient's health status and reduces healthcare costs. It has also been recognised as an independent function of the nursing profession and is a planned, 
systematic and logical process. ${ }^{711}$ Patient education is an important component of good quality healthcare. One of the teaching methods follows the ASSURE (Assessment, State objects, Select materials, , Require learner response, Evaluation) model, which provides guidelines to organise appropriate teaching for achieving health education. ${ }^{11} 1213$

Health insurance in Taiwan has nationwide coverage, which provides a comprehensive package of preventive measures and health services. ${ }^{14}$ It allows equal access to healthcare for all citizens by monitoring financial expenditures and improving healthcare outcomes. This high insurance rate results in an extremely high nurse-to-patient ratio in Taiwan compared with other countries and involves providing health education to patients when they are hospitalised. ${ }^{15}$ Providing patient education requires time based on patient's needs and involves one-on-one discussion. Also, in clinical practice, patients need repeated education.

Since the Electronic Signature Act was enacted in 2001, the process of maintaining electronic and digital health records has been rapidly developed. ${ }^{16} 17$ The legal elements and effects of electronic health records and signatures have become well-established under the act. These aspects have made electronic health records popular and have accepted and included safe and reliable internet, electronic records and unduplicated signatures. Moreover, identification of health providers involved in patient care would benefit communication among healthcare professionals. ${ }^{18} 19$ Therefore, electronic health records store patient data, strengthen record quality and enable interprofessional cooperation to achieve the goal of providing optimum patient healthcare.

Currently, health education is recorded under the content of caring activities in the nursing record section of the electronic health record. Records of patient education were not easy to identify in the document. Professional health providers in different units are unaware of the type of health education the patient or the patient's family has received. Furthermore, it is difficult to follow up patients' compliance with treatment after health education if the patient education details are not recorded. Thus, the development of a health education information system would fill the gap between the actual patient education and continued patient healthcare.

Developing a computerised system for patient education is not only to maintain a record but also to improve the working process of clinical practice, which includes appropriate methods, equipment and a computerised system for nurses. ${ }^{20}$ Developing an electronic patient education record system would be the last stage in completing nursing records digitally. ${ }^{21}$ Despite the benefit of storage space and the ease of transport, little is known about the effectiveness of documenting, nurses' satisfaction level and professional cooperation with the use of the Patient Education Assessment and Description Record System (PEADRS). ${ }^{22}$

\section{METHODS}

\section{Study aims}

The aim of this study is to develop a PEADRS and evaluate the effectiveness of the system on documenting, nurses' satisfaction level and professional cooperation. The study will be conducted in two phases. The first phase is to establish the PEADRS. In the second phase, a quasi-experimental study will be conducted to evaluate the effectiveness of the PEADRS. To investigate the outcomes, the following research questions will be asked:

1. How does the patient education care service influence the quality of patient education?

2. How does the PEADRS affect the length of nursing documentation and extent of completed patient education?

3. Is the satisfaction level of participants using the PEADRS different from those using traditional nursing records?

4. How does the PEADRS benefit professional cooperation?

\section{Study conceptual framework}

Converting the written records into electronic records (electronic health records) is a developmental approach in the field of medical science. It can transform patient healthcare management into knowledge and information sharing through the processes of information transferring, integrating, classifying and supporting decision making of patients and healthcare providers for enhancing patients' quality of healthcare. PEADRS provides a model that is based on the ASSURE teaching model (figure 1). The development process of the PEADRS includes technology, planning and management systems.

\section{Design}

The project involves a quasi-experimental design. The study will be conducted in two phases. In the first phase, the PEADRS will be established, developed and built. In the second phase, the effectiveness of the PEADRS will be evaluated, for which a non-synchronous design will be implemented. Subjects for the study will be recruited by random sampling of nurses providing education to patients admitted in a hospital or preparing for invasive examination or surgery in the general wards. The study will be conducted in a medical centre in central Taiwan. A total of 220 nurses will be recruited in each of the control group and the experimental group. The control group will use the traditional method of maintaining records in patient education, whereas the experimental group will apply the PEADRS for patient education and records.

\section{Independent variables}

To assess the effectiveness of the PEADRS in patient education related to those admitted for invasive examination or surgery preparation, the following outcomes will be measured:

1. PEADRS application: applying the ASSURE teaching model to the system. 


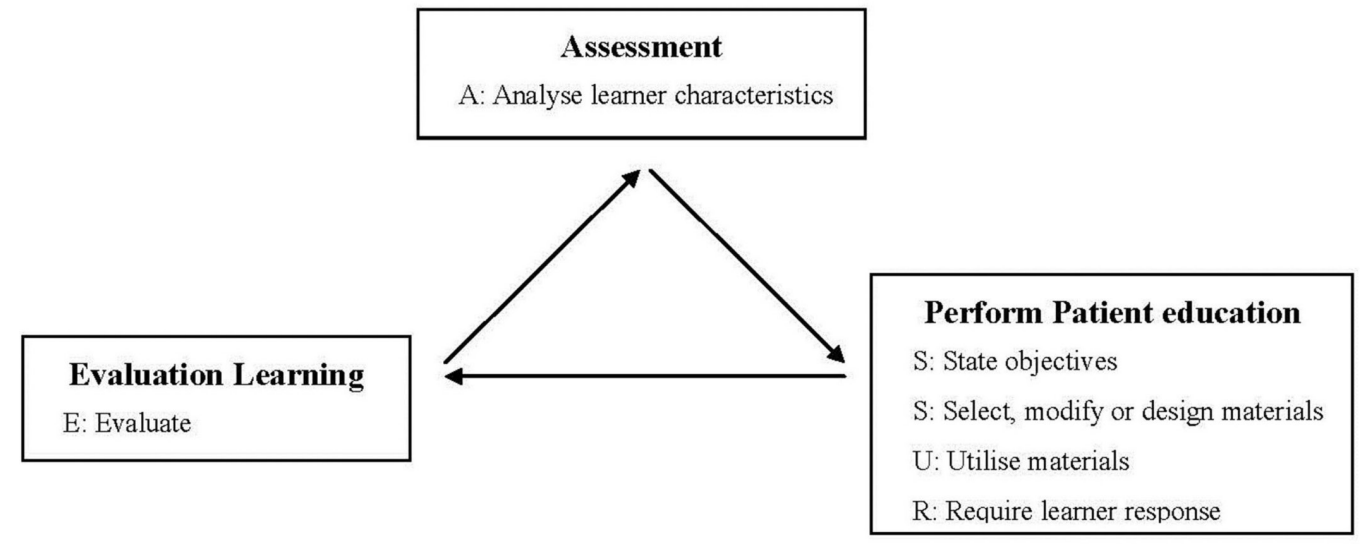

Figure 1 Structure of Patient Education Assessment and Description Record System based on ASSURE systematic teaching model.

2. The length of the nursing documentation process: observing and recoding the time taken by nurses in documenting health education.

3. Record the integrity of health education: assessing the completion rate of nurses' record.

4. Satisfaction level of nurses using the PEADRS: questionnaire to evaluate satisfaction levels in nurses using the PEADRS.

5. Interprofessional cooperation: is the PEADRS a platform for cognate professionals?

\section{Setting}

The study will be conducted in a medical centre in central Taiwan. The hospital is one of the nation's premier teaching hospitals with a capacity of 2111 beds. The hospital has 2980 health education materials in different languages, including brochures, posters and films. In the past decade, the average occupancy rate of hospitalised patients was $85 \%$ in this hospital. Many nurses are occupied in implementing health education and recording data.

\section{Participants}

The participants include nursing staff who will be recruited through random sampling from the medical centre in central Taiwan. The inclusion criteria are that they should be registered nurses, have a work experience of more than 3 months and should be working in a general ward. Participants will be excluded if they are working as assistant personnel. The optimal sample size was calculated using $G^{*}$ Power 3.1, with a mean difference of $\alpha=0.05$ and effect size of 0.2 in one sample case with pretest and post-test. Overall, 199 participants will be required to achieve a power of $80 \%$ to detect statistically significant differences. A total of 220 participants will be recruited, with $10 \%$ possibly missing in data collection. The same number of participants will be recruited for the control group. The control group will use the original method of recoding patient education in nursing records, and the experimental group will use the PEADRS.

To determine the effectiveness and stability of the PEADRS, the following procedures will be included: (1) the PEADRS interface design guidance to guide the operation of priority steps; (2) the consistency of data linking to the nursing care system, assisting nurses to determine patient facts and amendments; (3) set the content of professional care aspects and their description, meeting the needs of health education records; (4) the convenience for users (nurses); and (5) stability of informatics system. A pilot study was conducted to identify the barriers to and operation of using the PEADRS. A group of 30 nurses was recruited to make appropriate amendments.

\section{Patient and public involvement}

Patients and the public will not be involved in this study.

\section{Developing and establishing the PEADRS}

1. Establishing a system-developed project team: The team members include a primary investigator, two coprimary investigators (patient education committee chairman, director of nursing), two clinical health educators with more than 10 years of experience, five clinical nurses with 15 years of experience of working in a medical centre (internal medicine, surgery, obstetrics and gynaecology, paediatrics and emergency department), four senior technicians (internal medicine, surgery, rehabilitation and haemodialysis) and one informatics programmer. Several clinical observations of patient education are arranged for the informatics programmer to understand its implementation to reduce the cognitive gap and meet the needs of the users.

2. Regular meeting: The team meets twice a week for discussion. The nursing department is responsible for collecting information and setting up computer screen interfaces for the system and reviewing and analysing the current status of the implementation of the records. Relevant health education for different departments are encouraged by setting up procedures for patient education, including assessment and implementation, developing education content and documenting methods.

3. Setting up the projects: The system includes five professional care aspects: outpatient care, adult patient 


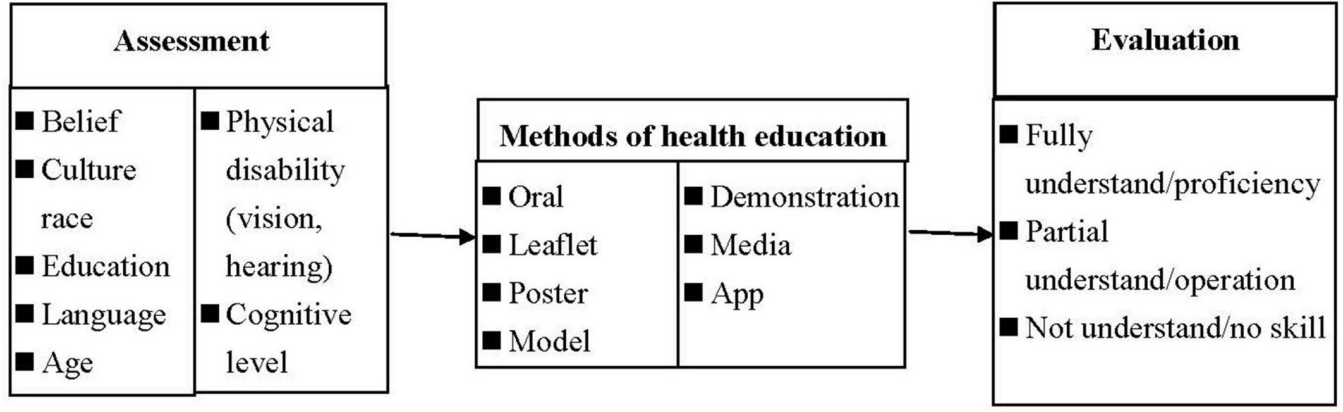

Figure 2 Patient Education Assessment and Description Record System design.

care, paediatric patient care, examination and treatment care and Chinese traditional medicine care. The content and procedure of health education can be divided into steps. For example, taking care of a wound would involve the following procedures: (1) keeping the wound clean and dry; (2) observing for signs of infection, including swelling, soreness and abnormal secretions; (3) taking wound-dressing precautions and knowing when to call for the nurse; (4) knowing how to use Steri-Strip/Adhesive Skin Closure; (5) using waterproof cover on the wound before shower; (6) having information regarding care of wound caused during removal of arterial catheter; and (7) knowing how to use Tegaderm Hydrocolloid Dressings.

4. Applying the ASSURE systematic teaching model: The process of ASSURE includes assessment prior to health education, implementation of health education and evaluation of health education on the functional interface of the PEADRS. The design and development of the assessment contents are based on the literature review and recommendations of clinical practice experiences and the experts' advices. It includes an assessment lens related to patient characteristics and the ability to learn, formulating the strategies to implement health education instruction and evaluating the learning outcome based on learners' understanding level (figure 2).

5. Operation system: The development of simple input interface was designed by the information programme engineer who also integrated the interface control software. To delaminate implementation of the ASSURE systematic teaching model, the following were the operation guidelines with priority steps: 'assessment of patients', 'implementation of health education' and 'evaluation of learning'. The components embedded into the design along with health education items allow addition of all types of special components or integration of system functional elements. To simplify the process, after clicking on the data entry, 'Nursing Integration System' of the 'assessment of patient', this will be linked to 'electronic health record system'. All functions in the Nursing Integrated System automatically generate patient records, resulting in the associated data, functional window prompts and feedback. Nurses in different duty shifts can use the 'inquiry' function to find any related health information and know about the patient/family members who have received health education and the type of health education.

6. Confidentiality and stability: According to the regulation of electronic medical records, the establishment of information management system will ensure the stability, reliability, system confidentiality and security of the overall system operation.

\section{Instruments}

Several methods will be used to evaluate the study outcomes, including clinical data, nursing record review, observation, interview and questionnaire. To ensure reliable and unbiased extraction of data from the observation, research assistants will be trained in observing, recording, interviewing and accounting data. Inter-rater reliability of the study will be noted.

A self-report questionnaire will help to measure the satisfaction level of nurses using the PEADRS. Questions are based on the users' adoption and implementation of the PEADRS. The questionnaire includes 12 questions that are answered on a five-point Likert scale, ranging from 'very good' to 'very bad'. To have an in-depth understanding of the constructive views of nurses, their attitude and their demands, two open-ended questions are added: (1) enhancing factors in adopting the PEADRS; and (2) impeding factors in adopting the PEADRS. The instrument's readability, accuracy and adaptability will be determined by panel of experts and a pilot study. Face validity will be determined by expert review with a CVI (Content Validity Index) calculation. Reliability will be tested for reliable internal consistency by a pilot study with a sample of 30 participants.

Professional cooperation will be measured by the PEADRS. The system would provide the choice of a date range (seven or all), on which health education items implemented through the system can be queried across departments (professionals). Nurses can choose the 'query' option to understand what type of information was already provided to the patient/family members and can continue to follow up on their response (reaction/ adherence). Thus, the system will record the times when patients visit different professionals (nurses/technician/ pharmacist/etc) and have health education connected in 
time and completed. Also, the questions about satisfaction with professional cooperation is also provided in the instrument. For the control group, the professional cooperation can be observed and counted in the traditional nursing records.

\section{Data collection and analysis}

After the PEADRS is established, baseline data regarding the time of implementing health education and record will be collected, for which a total of 220 nurses providing health education to inpatients with various invasive examination and surgery will be enrolled. The time taken by the nurses for documenting patient education will be observed and recorded. Data on the satisfaction level of nurses using the PEADRS will be collected using a questionnaire. Also, data on interviewing nurses' perspective on applying the PEADRS will be gathered. The results of professional cooperation will be measured by checking the record on the PEADRS and the questionnaire.

The data will be analysed using descriptive and inferential statistics. To evaluate the PEADRS, the McNemar and paired $t$ tests will be used to calculate the means of length of the nursing documentation between pretest and post-test responses. Next, a t test will be conducted to compare the two groups in recording the integrity of health education, satisfaction level and interprofessional cooperation. An alpha level of 0.05 will be considered statistically significant.

\section{Ethics and dissemination}

Procedure for protecting individual information confidentiality will be followed by electronic medical record management practices of hospitals in accordance with the national medical law and personal data protection law. Ethical approval was obtained. The study excludes all individual identification of participants or demographics data. Participants' privacy will be protected according to Electronic Medical Record Management Practices of the hospital.

\section{DISCUSSION}

Informatics systems have contributed to healthcare. ${ }^{16}$ The development and application of the PEARDS is an important stage in promoting a smarter medical care. It is the first act in integrating health education resources into a computerised system in electronic medical records. The study is expected to achieve the following: (1) establishing an assessment, description and record informatics system on patient education for clinical practice in electronic medical records; (2) guiding nurses to provide patient education with the ASSURE systematic teaching model. The results of this study will help nurses to assess the needs of patients/family members for education, such as physical and psychological barrier and emotional status. Thus, it would help nurses to choose an appropriate method for the learner based on assessment and evaluation of learning outcomes; (3) enabling nurses, easily, to review patient data related to health education, which will not only reduce repetition of teaching but also emphasise the evaluation of learning to provide efficacious education. The nurses will recognise the type of patient education that patients/family members have already received, thus facilitating coordination, communication and cooperation between healthcare providers. This would reduce the transmission time and help follow up patient's adherence so that the patient could enjoy continuity and integrity of healthcare; (4) improving data consistency, recording integrity and minimising storage space for medical records; and (5) facilitating functional window prompts and feedback. The records cannot be saved until the data entry is completed; this improves the completion of patient education, which helps improve quality of care.

\section{Limitations}

Although the steps of the research process have been followed, there are limitations based on staffing, time and economic constraints: (1) education is a highly complex activity, which combines interactive situations with humanities, ethics and educational context. The relationship between the nurses and learners is subjective. The experimental research emphasises standardisation and repeated verification, but education is often unique and cannot be repeated; hence, the cause and effect in health education still has limitations; (2) the study will involve conducting a survey on the nurses' implementation of patient education and digital record, as a representative of patients with invasive procedures or examinations and operation; thus, the results of this study may not be generalised to different types of patient education; (3) although the nurses are willing to cooperate with the implementation and response, the variation in quality, teaching ability and system operation skills may have an impact on the effectiveness of the validity of the new system.

\section{CONCLUSION}

This study aims to study whether the PEADRS has a positive effect on the quality of healthcare. This study develops a PEADRS, which could help promote the quality of clinical practice. There is a significant gap in the electronic health record information system. The quality of the caring process in patient education documentation is evident. The teaching models such as assessment, teaching methods and evaluation of learning provide a guideline for nurses in patient education, which enhances the nurses' capability in health education. A study of nurse end users of the PEADRS should be conducted for yielding more information about barriers, frustrations, quality needs and preferences of nursing staff. Also, a further study is needed to study the effect of the PEADRS on patient outcomes such as quality of life, medication adherence). A follow-up study is planned to assess effects of user-designed system changes based on the results of this study. Further work following PEADRS improvements will explore the satisfaction of paramedical 
staff, including teamwork cooperation, integrated care and sustainability. This study will provide new information specific to patient education and will assist in providing an evidence base for this innovation at a level that has not yet been established.

Contributors $\mathrm{C}$-HS is the principal investigator of the PEADRS. Study concept was given by C-HS, Y-SC and D-YC. C-HS, T-CL, W-FM, T-HL, Y-SC and L-CH contributed to the design of the study and/or to the implementation of the Intervention. C-HS and Y-SC wrote the manuscript and L-CH revised for important intellectual contents. All authors approved the final manuscript.

Funding We would like to thank the China Medical University Hospital, Taiwan, for financial support (DMR-106-143, DMR-107-141).

Competing interests None declared.

Patient consent Not required.

Ethics approval The ethical approval has been approved by the Institutional Review Board of China Medical University Hospital (CMUH107-REC2-024).

Provenance and peer review Not commissioned; externally peer reviewed.

Open Access This is an Open Access article distributed in accordance with the Creative Commons Attribution Non Commercial (CC BY-NC 4.0) license, which permits others to distribute, remix, adapt, build upon this work non-commercially, and license their derivative works on different terms, provided the original work is properly cited and the use is non-commercial. See: http://creativecommons.org/ licenses/by-nc/4.0/

(C) Article author(s) (or their employer(s) unless otherwise stated in the text of the article) 2018. All rights reserved. No commercial use is permitted unless otherwise expressly granted.

\section{REFERENCES}

1. Biron P, Metzger MH, Pezet C, et al. An information retrieval system for computerized patient records in the context of a daily hospital practice: the example of the Léon Bérard Cancer Center (France). Appl Clin Inform 2014;5:191-205.

2. Natarajan $\mathrm{K}$, Stein $\mathrm{D}$, Jain $\mathrm{S}$, et al. An analysis of clinical queries in an electronic health record search utility. Int $J$ Med Inform 2010;79:515-22.

3. Carrington JM, Effken JA, Fann F. Strengths and limitations of the electronic health record for documenting clinical events. Comput Inform Nurs 2011;29:360-7.

4. Peltonen LM, Alhuwail D, Ali S, et al. Current Trends in Nursing Informatics: Results of an International Survey. Stud Health Technol Inform 2016;225:938-9.

5. Jarvis B, Johnson T, Butler P, et al. Assessing the impact of electronic health records as an enabler of hospital quality and patient satisfaction. Acad Med 2013;88:1471-7.
6. Jones J, Schilling K, Pesut D. Barriers and benefits associated with nurses information seeking related to patient education needs on clinical nursing units. Open Nurs J 2011;5:24-30.

7. Falvo D. Effective Patient Education: A Guide to Increased Adherence. Sudbury: Jones \& Bartlett Learning, 2010:461.

8. Berkman ND, Sheridan SL, Donahue KE, et al. Low health literacy and health outcomes: an updated systematic review. Ann Intern Med 2011;155:97-107.

9. Inzucchi SE, Bergenstal RM, Buse JB, et al. Management of hyperglycemia in type 2 diabetes, 2015: a patient-centered approach: update to a position statement of the American Diabetes Association and the European Association for the Study of Diabetes. Diabetes Care 2015;38:140-9.

10. Marcus C. Strategies for improving the quality of verbal patient and family education: a review of the literature and creation of the EDUCATE model. Health Psychol Behav Med 2014;2:482-95.

11. Bergh AL, Karlsson J, Persson E, et al. Registered nurses' perceptions of conditions for patient education - focusing on organisational, environmental and professional cooperation aspects. J Nurs Manag 2012;20:758-70.

12. Branch RM, Kopcha TJ. Instructional design models form handbook of research on educational communications and technology. New York: Springer, 2014:77-87.

13. Bastable SB. Essentials of patient education. Sudbury (MA): Jones \& Bartlett Learning, 2008:203.

14. Lee YC, Huang YT, Tsai YW, et al. The impact of universal National Health Insurance on population health: the experience of Taiwan. BMC Health Serv Res 2010;10:225.

15. Liang YW, Chen WY, Lee JL, et al. Nurse staffing, direct nursing care hours and patient mortality in Taiwan: the longitudinal analysis of hospital nurse staffing and patient outcome study. BMC Health Serv Res 2012;12:44.

16. Bowman S. Impact of electronic health record systems on information integrity: quality and safety implications. Perspect Health Inf Manag 2013;10:1-13.

17. Victoroff Md MS. Electronic health records: what does your signature signify? Patient Saf Surg 2012;6:20.

18. Cho I, Kim E, Choi WH, et al. Comparing usability testing outcomes and functions of six electronic nursing record systems. Int $J$ Med Inform 2016;88:78-85.

19. Varpio L, Rashotte J, Day K, et al. The EHR and building the patient's story: A qualitative investigation of how EHR use obstructs a vital clinical activity. Int J Med Inform 2015;84:1019-28.

20. Ratwani RM, Fairbanks RJ, Hettinger AZ, et al. Electronic health record usability: analysis of the user-centered design processes of eleven electronic health record vendors. J Am Med Inform Assoc 2015;22:1179-82.

21. Sittig DF, Wright A. What makes an EHR "open" or interoperable?: Table 1:. Journal of the American Medical Informatics Association 2015;22:1099-101.

22. Topaz M, Ronquillo C, Peltonen LM, et al. Nurse Informaticians Report Low Satisfaction and Multi-level Concerns with Electronic Health Records: Results from an International Survey. AMIA Annu Symp Proc 2016;2016:2016-25. 